\title{
Qualidade do percolado de solos que receberam vinhaça em diferentes doses e tempo de incubação ${ }^{1}$
}

\author{
Fabio L. Brito ${ }^{2}$, Mario M. Rolim ${ }^{2}$, Jose A. A. da Silva ${ }^{3}$ \& Elvira M. R. Pedrosa ${ }^{2}$
}

\begin{abstract}
RESUMO
Neste estudo, objetivou-se avaliar a qualidade do percolado de solos que receberam doses de vinhaça em diferentes tempos de incubação. Para isto, foram reproduzidos, em 27 colunas de PVC de $20 \times 110 \mathrm{~cm}$ (diâmetro x altura) solos classificados como Nitossolo Háplico, Argissolo Amarelo e Espodossolo Cárbico, com horizontes, espessuras e densidades semelhantes aos do solo original. Os solos foram tratados com vinhaça em doses equivalentes a 0 (Testemunha), 350 e $700 \mathrm{~m}^{3}$ ha $^{-1}$ submetidos aos tempos de incubação de 30 e 60 dias. Os efluentes coletados foram analisados quanto a $\mathrm{DBO}_{5}, \mathrm{DQO}, \mathrm{CE}, \mathrm{SDT}$ e pH. Os resultados indicaram que as variações verificadas nos parâmetros analisados, decorrentes da aplicação da vinhaça ao solo, não trazem maiores preocupações quanto às questões ambientais visto que, quando comparados os valores do percolado com os da vinhaça in natura, os solos, apesar de apresentarem diferentes texturas e, conseqüentemente, diferentes propriedades físico-químicas, mostraram elevado poder de retenção, diminuindo sobremaneira a possibilidade do resíduo causar poluição às águas subsuperficiais.
\end{abstract}

Palavras-chave: aproveitamento de resíduos, cana-de-açúcar, qualidade ambiental

\section{Quality of percolate from soils after application of different doses and incubation time of vinasse}

\begin{abstract}
The objective of this study was to evaluate the quality of percolation from three soil classes that received vinasse doses with different incubation times. The soils, classified as Haplic Nitosol, Yellow Argisol and Carbic Spodosol, were reproduced in twenty-seven $20 \times 110 \mathrm{~cm}$ PVC columns (diameter $\times$ height) with horizons, thickness and densities similar to original soil. The soils were treated with vinasse doses equivalent to 0 (control), 350 and $700 \mathrm{~m}^{3}$ ha $^{-1}$, and submitted to 30 and 60 days incubation. The collected effluents were analyzed for $\mathrm{BOD}_{5}, \mathrm{COD}, \mathrm{EC}$, TDS and $\mathrm{pH}$. The results indicated that variations in the analyzed parameters, due to vinasse application in the soil, do not bring concerns in relation to environmental quality. Despite different textures and consequently, different physiochemical properties, the comparison of percolates with natural vinasse data indicated that the soils presented high retention power, reducing the possibility of this waste causing pollution to the subsurface waters.
\end{abstract}

Key words: residue use, sugarcane, environment quality

\footnotetext{
Parte da Dissertação de Mestrado do primeiro autor apresentada ao Programa de Pós-Graduação em Ciência do Solo, UFRPE

2 DTR/UFRPE. Rua Dom Manoel de Medeiros s/n, Dois Irmãos, CEP 52171-900, Recife, PE. Fone: (81) 3302-1276. E-mail: rolim@ufrpe.br; epedrosa@ufrpe.br

DCF/UFRPE. Fone: (81) 3302-1288. E-mail: jaaleixo@uol.com.br
} 


\section{INTRODUCÃO}

A vinhaça é o principal resíduo líquido da indústria sucroalcooleira e apresenta, na sua composição, quantidade significativa de elementos químicos e de matéria orgânica essencial à planta. Outrora, a vinhaça era lançada, na forma bruta, nos cursos de água, desencadeando uma série de fatores adversos no que diz respeito às questões ambientais; posteriormente, passou a ser lançada no solo, na forma in-natura, o que provocou, de início, muitos questionamentos sobre os efeitos no solo e no lixiviado. Várias pesquisas (Ferreira \& Monteiro, 1987; Camargo et al., 1983; RIDESA, 1994; entre outras), indicaram os benefícios do aproveitamento desse resíduo na fertirrigação como fonte de potássio, matéria orgânica, sobretudo a não poluição dos cursos de água.

Desenvolveram-se, a partir da década de oitenta, estudos sobre a possibilidade da vinhaça originar poluição nas águas subsuperficiais porém essas investigações foram desenvolvidas unicamente com a visão de se avaliar o potencial poluidor desse resíduo (Cunha et al., 1987; Casarini et al., 1987; Gloeden et al., 1990); contudo, RIDESA (1994) observou que a questão da aplicação de vinhaça ou de qualquer água residuária no solo deve centrar-se na hipótese do solo, em sua zona não saturada e se comportar como verdadeiro sistema de tratamento, apresentando grande capacidade de remoção dos componentes da vinhaça.

Paganini (1997) estudou a capacidade de redução da demanda bioquímica de oxigênio no quinto dia $\left(\mathrm{DBO}_{5}\right)$, doravante denominada DBO, do lixiviado de um solo incorporado com lodo de esgoto e explica que a oxidação biológica é o principal mecanismo de remoção da matéria orgânica, no caso específico solúvel no esgoto e, ainda, que a matéria orgânica coloidal é responsável por cerca de 50\% da carga da DBO do resíduo.

Por sua vez, Ludovice (1997) trabalhou no monitoramento de efluente de canal não revestido para condução de vinhaça no qual utilizou, como parâmetros de avaliação, as análises de $\mathrm{pH}$, condutividade elétrica (CE), DBO e demanda química de oxigênio (DQO) entre outras, verificando que, ao longo dos anos, a taxa de remoção, ou seja, a capacidade do solo em reter as várias substâncias presentes na vinhaça, não foi eficiente apresentando, no caso específico, efeito poluente sobre o lençol freático.

Por outro lado, em experimento de campo, Lyra et al. (2003) trabalharam em uma área de 12 ha, fertirrigada com vinhaça, na qual foram instalados 30 poços de monitoramento com profundidade de $3 \mathrm{~m}$, para avaliar a qualidade da água do lençol freático, concluindo que o poder de remoção do solo nas condições estudadas, para as variáveis DBO e DQO, foi satisfatória, indicando a eficiência da redução desses parâmetros.

Apesar da grande produção de conhecimento relativo a estudos da aplicação de vinhaça e respectivos efeitos nos atributos do solo (Camargo et al., 1988) e na produção da cana-de-açúcar (Orlando Filho et al., 1983), só na década de noventa se iniciaram os estudos com vinhaça, voltados para a questão ambiental (RIDESA, 1994). Pesquisas que possam consolidar a utilização da vinhaça na fertirrigação da cana-de-açúcar como prática viável e ambientalmente segura, são fundamentais para aperfeiçoamento do sistema de produção sucroalcooleiro.

Objetivou-se, dentro deste contexto, quanto ao estudo, avaliar a qualidade do lixiviado em três tipos de solo que receberam doses de vinhaça com diferentes tempos de incubação verificando o potencial de poluição ao lençol freático.

\section{MATERIAL E MÉTODOS}

O experimento foi conduzido no Laboratório de Mecânica dos Solos e Aproveitamento de Resíduos e as análises realizadas nos Laboratórios de Química do Solo, Fertilidade do Solo e Física do Solo da UFRPE, entre os meses de janeiro a março de 2004.

A vinhaça utilizada foi proveniente da Usina Petribu, Lagoa de Itaenga, PE, coletada na tubulação de saída da destilaria. A caracterização físico-química da vinhaça utilizada encontra-se na Tabela 1.

Tabela 1. Caracterização físico-química da vinhaça in natura utilizada no presente estudo

\begin{tabular}{lcc}
\hline Variáveis & Unidade & Valor \\
$\mathrm{pH}$ & - & 4,4 \\
$\mathrm{DBO}$ & $\left(\mathrm{mg} \mathrm{L}^{-1}\right)$ & 5.000 \\
$\mathrm{DQO}$ & $\left(\mathrm{mg} \mathrm{L}^{-1}\right)$ & 26.771 \\
$\mathrm{CE}$ & $\left(\mathrm{dS} \mathrm{m}^{-1}\right)$ & 11,5 \\
$\mathrm{SDT}$ & $\left(\mathrm{mg} \mathrm{L}^{-1}\right)$ & 11.352 \\
$\mathrm{~K}$ & $\left(\mathrm{mg} \mathrm{L}^{-1}\right)$ & 1.123 \\
$\mathrm{Ca}$ & $\left(\mathrm{mg} \mathrm{L}^{-1}\right)$ & 352 \\
$\mathrm{Mg}$ & $\left(\mathrm{mg} \mathrm{L}^{-1}\right)$ & 16 \\
$\mathrm{Na}$ & $\left(\mathrm{mg} \mathrm{L}^{-1}\right)$ & 113 \\
\hline * & &
\end{tabular}

* SDT - sólidos dissolvidos totais

Utilizaram-se três tipos de solo representativos da zona da mata canavieira do Estado de Pernambuco, classificados como: Espodossolo Cárbico Órtico Duripânico Arênico de textura arenosa, proveniente da Unidade de Execução de Pesquisa de Itapirema, Goiana, PE; Nitossolo Háplico Eutrófico típico, textura argilosa, coletado no Engenho Vazante (Usina Aliança) Aliança, PE, e Argissolo Amarelo Distrófico fragipânico, textura média/argilosa, coletado no Sítio Santa Luzia, Aliança, PE. Na Tabela 2 encontram-se os resultados da análise física dos solos utilizados.

Os solos foram reproduzidos em 27 colunas de PVC de $20 \mathrm{~cm}$ de diâmetro por $110 \mathrm{~cm}$ altura, onde foram simuladas as condições de campo até a profundidade de $100 \mathrm{~cm}$, respeitando-se as densidades e profundidades de cada horizonte do solo.

Antes de receberem vinhaça, as colunas de solo foram saturadas através de ascensão da água, pela parte inferior, local da instalação dos drenos, e posteriormente liberadas, permitindo-se escoar o excesso de água até estancamento atingindo, conseqüentemente, a máxima capacidade de retenção de água contra a ação da gravidade, equivalente à capacidade de campo (Cambuim, 1983). 
Tabela 2. Caracterização física dos solos utilizados no presente estudo

\begin{tabular}{|c|c|c|c|c|c|c|}
\hline \multirow{2}{*}{ Hor.* } & \multirow{2}{*}{$\begin{array}{l}\text { Prof..* } \\
\text { (cm) }\end{array}$} & Ds & Dp & Areia & Silte & Argila \\
\hline & & \multicolumn{2}{|c|}{$\left(\mathrm{g} \mathrm{cm}^{-3}\right)$} & \multicolumn{3}{|c|}{$\left(g_{\mathrm{kg}}-1\right)$} \\
\hline \multicolumn{7}{|c|}{ Nitossolo } \\
\hline Ap & $0-15$ & 1,46 & 2,86 & 340 & 360 & 300 \\
\hline$B_{1} t$ & $15-35$ & 1,34 & 2,9 & 240 & 330 & 430 \\
\hline$B_{21} t$ & $35-70$ & 1,29 & 2,86 & 180 & 300 & 520 \\
\hline$B_{22} \mathrm{t}$ & $70-100$ & 1,38 & 2,86 & 210 & 300 & 490 \\
\hline \multicolumn{7}{|c|}{ Argissolo } \\
\hline$A p$ & $0-28$ & 1,51 & 2,74 & 792 & 73 & 135 \\
\hline$E$ & $28-50$ & 1,63 & 2,86 & 795 & 57 & 149 \\
\hline EB & $50-87$ & 1,64 & 2,73 & 688 & 40 & 272 \\
\hline $\mathrm{Btx} / \mathrm{E}$ & $87-100$ & 1,50 & 2,71 & 468 & 93 & 439 \\
\hline \multicolumn{7}{|c|}{ Espodossolo } \\
\hline Ap & $0-15$ & 1,86 & 2,57 & 895 & 40 & 65 \\
\hline E1 & $15-46$ & 1,87 & 2,67 & 921 & 34 & 46 \\
\hline E2 & $46-90$ & 1,82 & 2,73 & 906 & 30 & 65 \\
\hline $\mathrm{Bh}$ & $90-100$ & 1,64 & 2,67 & 905 & 37 & 58 \\
\hline
\end{tabular}

Posteriormente, as colunas foram submetidas a aplicações de doses equivalentes à Testemunha - Testemunha (água), 350 (Tratamento 1) e 700 (Tratamento 2) $\mathrm{m}^{3} \mathrm{ha}^{-1}$ de vinhaça, totalizando três tratamentos, para dois períodos de incubação respectivamente, 30 e 60 dias, conduzidos com três repetições. Após cada período de incubação aplicou-se uma lâmina de lixiviação (água) equivalente ao volume de poros de cada solo, contidos nas respectivas colunas e se coletou o percolado (duas vezes, 30 e 60 dias, na mesma coluna) através dos drenos devidamente colocados na parte inferior das colunas.

Após coleta e homogeneização de todo efluente percolado, alíquotas para determinações físico-químicas das variáveis DBO, DQO, CE, SDT e $\mathrm{pH}$ foram retiradas, segundo o Standard Methods for Examination of Water and Wastewater (APHA, 1995).

O delineamento experimental utilizado foi inteiramente casualizado e os tratamentos foram distribuídos em arranjo fatorial, 3 tipos de solo (Espodossolo Cárbico Órtico, Nitossolo Háplico Eutrófico e Argissolo Amarelo Distrófico) $\times 3$ taxas de aplicação de vinhaça $\left(0,350\right.$ e $\left.700 \mathrm{~m}^{3} \mathrm{ha}^{-1}\right)$, com medidas repetidas ao longo do tempo (30 e 60 dias). Para análise estatística dos dados aplicou-se o teste de esfericidade de Mauchly (Xavier, 2000) para definição do tipo de análise a ser utilizada: parcelas subdivididas, caracterizada pela independência das duas medidas ao longo do tempo, ou multivariada de medidas repetidas, em que se considera a dependência entre as medidas. Utilizou-se o software estatístico SAS (Statistical Analysis System), com níveis de significância de 5\% para o teste de $\mathrm{F}$ e as médias foram comparadas pelo teste de Tukey, a 5\% de significância.

\section{RESULTADOS E DISCUSSÃO}

O teste de esfericidade de Mauchly indicou, para o presente estudo, o uso de análise multivariada de medidas repetidas, para todas as variáveis estudadas.

A análise multivariada, a nível de $5 \%$ de probabilidade aplicada à variável DBO, mostrou diferença significativa para $\mathrm{o}$ efeito do solo, dose, tempo e interações tempo $\times$ solo e solo $\times$ dose, mas não significativa para as interações tempo $\times$ dose de aplicação de vinhaça e tempo $\times$ solo $\times$ dose. Os menores $(\mathrm{P} \leq 0,05)$ valores de DBO ocorreram no percolado do Nitossolo nos dois períodos estudados (Figura 1A e B). Diferenças significativas entre Argissolo e Espodossolo foram detectadas aos 60 dias com os maiores valores de DBO no Espodossolo (Figura 1B).

A taxa de remoção da DBO obedeceu a uma seqüência esperada (Ludovice, 1997), considerando-se o teor de argila dos solos, visto que o Nitossolo que apresenta o maior teor de argila entre os demais, foi mais eficiente que o Argissolo que, por sua vez, foi superior ao Espodossolo de textura mais arenosa. O fato do Argissolo e do Esposossolo não diferirem aos 30 dias (Figura 1A) pode ser explicado pela presença do horizonte espódico, de acúmulo de matéria orgânica no Espodossolo, que pode ter favorecido o acúmulo da matéria orgânica presente na vinhaça, principal material responsável pela elevada DBO do resíduo. Brito et al. (2003), também em experimento com Espodossolo, encontraram resultados semelhantes.

Com relação à possível variação da DBO no percolado, devido às diferentes doses, constatou-se que, mesmo para a maior dose, os solos tiveram elevada capacidade de reter a matéria orgânica existente no resíduo, atingindo valores semelhantes aos encontrados na Testemunha que só recebeu água.

A taxa de remoção para a DBO, após 30 dias de incubação, chegou a 99,96\% na maior remoção encontrada, comparando-se o percolado do Nitossolo na menor dose de resíduo com a vinhaça in-natura e 99,81\% de remoção para a menor taxa encontrada, contrastando-se a vinhaça com o percolado do Argissolo na dose de $700 \mathrm{~m}^{3} \mathrm{ha}^{-1}$ do resíduo; valores semelhantes foram encontrados aos 60 dias; esses resultados condizem com o observado por Paganini (1997) sobre a eficiência da biodegradação da matéria orgânica no solo, ocasionada pela combinação de processos físicos, químicos e biológicos.

Efeitos significativos na DQO dos percolados ocorreram em relação ao solo, dose, tempo e interações solo $\times$ dose, tempo $\times$ solo, tempo $\times$ taxa de aplicação do resíduo, e não significativo para a interação tempo $\times$ solo $\times$ dose. Semelhante aos níveis de $\mathrm{DBO}$, os menores $(\mathrm{P} \leq 0,05)$ valores de DQO ocorreram em Nitossolo nos dois períodos estudados, entretanto, não se detectaram diferenças significativas entre Argissolo e Espodossolo aos 30 e 60 dias. Ao contrário da DBO, os valores de DQO variaram com a taxa de vinhaça aplicada. Aos 30 e 60 dias, a DQO em solos não tratados com o resíduo foi significativamente menor que em solos tratados porém não houve diferença entre solos tratados com 350 e $700 \mathrm{~m}^{3}$ ha $^{-1}$ (Figura 1C e D).

A análise multivariada da CE indicou efeito significativo do solo, dose, tempo e todas as interações, exceto tempo $\times$ solo $\times$ dose de vinhaça aplicada. As menores $(\mathrm{P} \leq 0,05)$ CE ocorreram em percolados de Nitossolo mas não houve diferença significativa entre percolados do Argissolo e do Espodossolo, para os dois períodos de incubação estudados 
(Figura 1E e F). Os resultados indicam que o solo com maior teor de argila (Nitossolo) indicou maior capacidade de reter, no perfil, os elementos responsáveis pela elevação da CE do efluente, corroborando com a afirmação de Sengik et al. (1988) de que as respostas nos solos que recebem vinhaça dependem de suas características físicas,
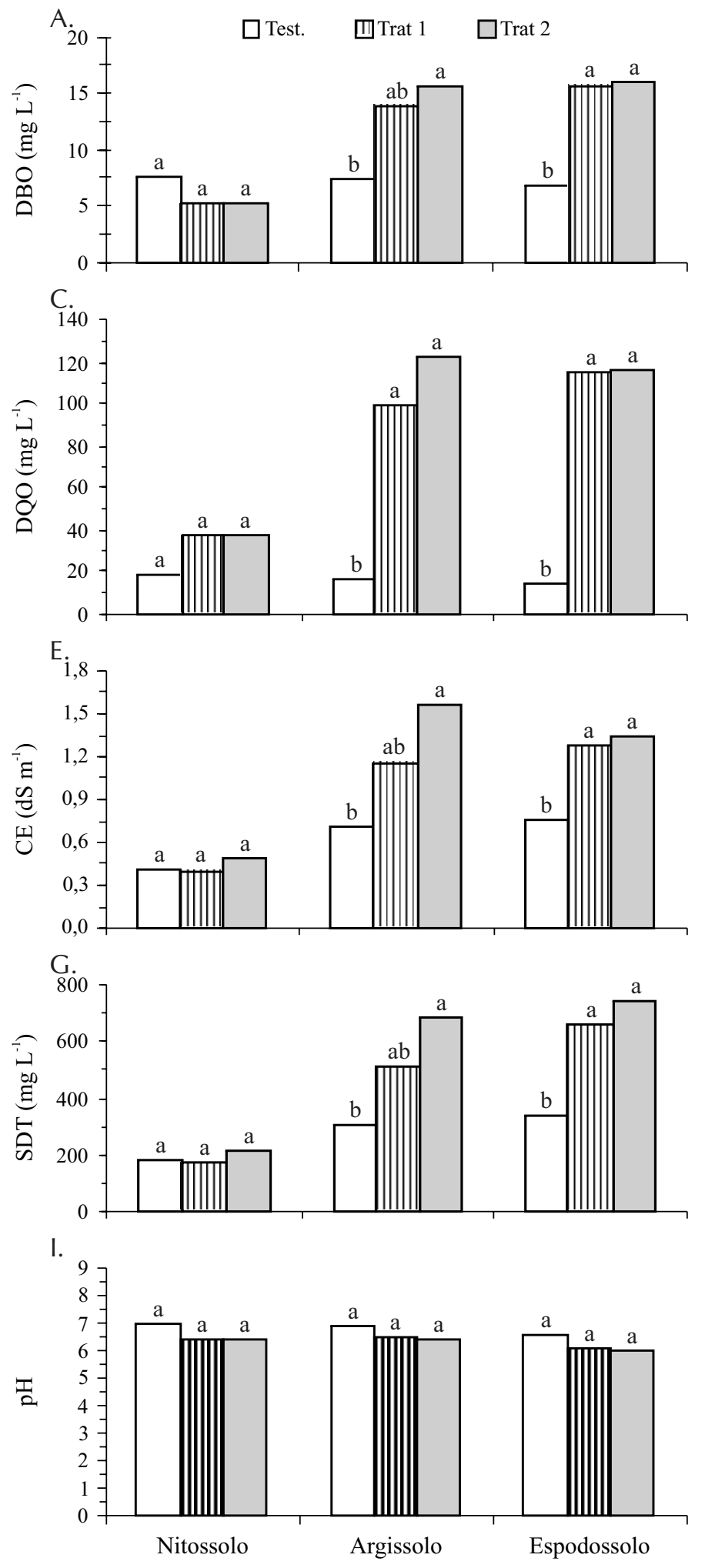

conseqüentemente, as respostas dos percolados também.

Ainda com relação à taxa de remoção dos diferentes tipos de solo, a CE apresentou inversão do Argissolo que, para esta variável, mostrou maior concentração que o Espodossolo, mesmo que, estatisticamente, não tenha sido diferente, quando comparada com a seqüência esperada em relação ao

B.

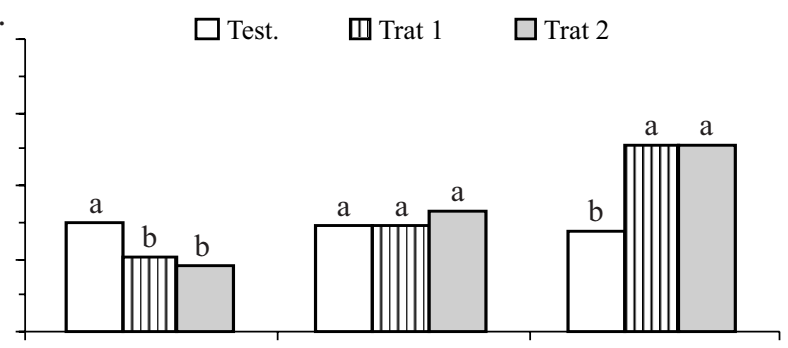

D.

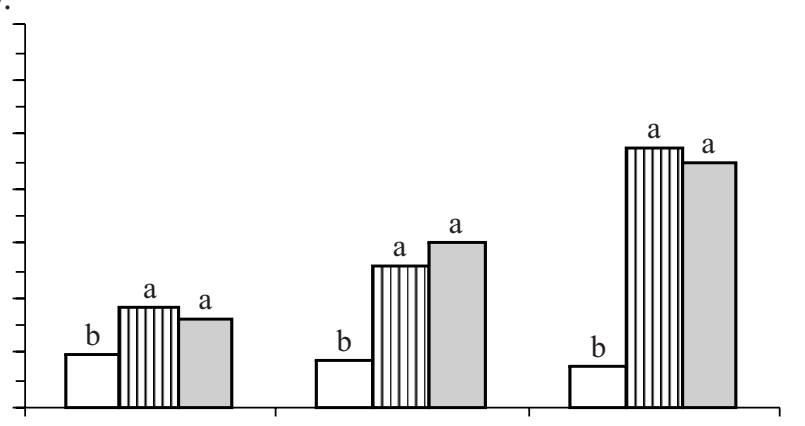

F.

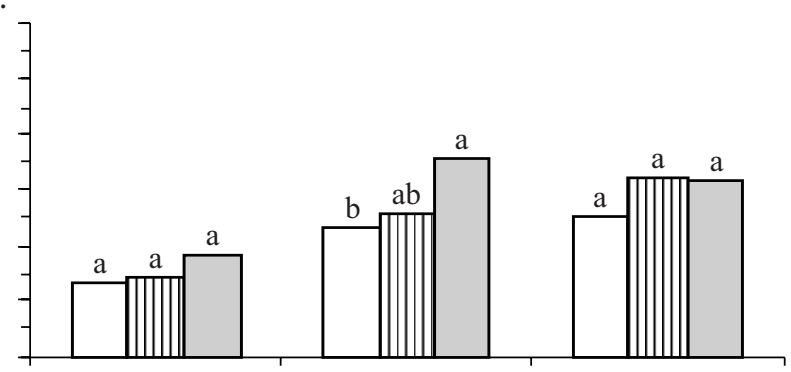

$\mathrm{H}$.

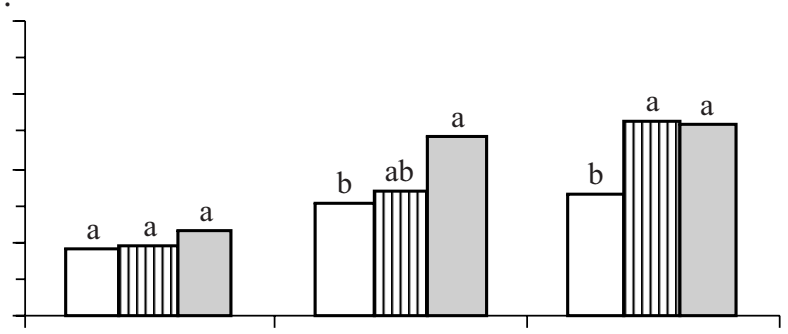

J.

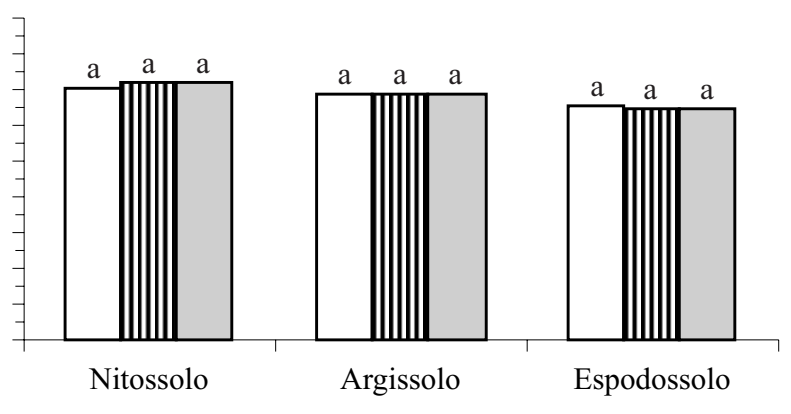

Figura 1. $\mathrm{DBO}(\mathrm{A}$ e B), $\mathrm{DQO}(\mathrm{C}$ e D), $\mathrm{CE}(\mathrm{E}$ e F), $\mathrm{SDT}(\mathrm{G} \mathrm{e} \mathrm{H)}$, e pH (l e J), do percolado dos três tipos de solo aos 30 e 60 dias após a aplicação de vinhaça (médias seguidas de mesma letra não diferem pelo teste de Tukey, a $5 \%$ de probabilidade) 
teor de argila, indicando que, para esta variável, a presença do horizonte espódico no Espodossolo foi o principal responsável pela redução da CE no percolado porém mesmo o Argissolo na dose mais elevada obteve uma taxa de remoção para a variável bastante alta, ficando na ordem de 86,41\%. Esses resultados estão de acordo com os encontrados por Brito et al. (2003).

Aos 30 dias (Figura 1E), a CE de percolados de solos não tratados foi menor $(\mathrm{P} \leq 0,05)$ que em solos tratados mas não ocorreu diferença entre os tratamentos com 350 e $700 \mathrm{~m}^{3} \mathrm{ha}^{-1}$; ao contrário, a CE do percolado dos solos que receberam $350 \mathrm{~m}^{3} \mathrm{ha}^{-1}$ de vinhaça diferiu significativamente dos que receberam $700 \mathrm{~m}^{3}$ ha $^{-1}$ mas não da Testemunha, aos 60 dias.

A variável SDT apresentou respostas significativas para todos os efeitos e interações testados, exceto para solo $\times$ dose, aos 60 dias. Embora o SDT tenha sido significativamente menor em Nitossolo, aos 30 e 60 dias, diferenças significativas entre Argissolo e Espodossolo só foram observadas aos 60 dias com as maiores concentrações verificadas no Espodossolo. Os resultados do SDT do efluente dos três tipos de solo aos $30(\mathrm{G})$ e $60(\mathrm{H})$ dias de incubação, após a aplicação de vinhaça, estão apresentados na Figura $1 \mathrm{G}$ e H.

De acordo com a Figura $1 \mathrm{G}$ e $\mathrm{H}$, todos os solos tiveram elevado poder de retenção, visto que a variação dos resultados encontrados nos percolados, 162; 211; 510; 689; 254,66; e 269,33 mg L-1 para Nitossolo, Argissolo e Espodossolo nas doses 350 e $700 \mathrm{~m}^{3} \mathrm{ha}^{-1}$ respectivamente, foi extremamente menor que a encontrada na vinhaça in-natura $11.352 \mathrm{mg} \mathrm{L}^{-1}$; além disso e segundo Ayers \& Westcot (1991), o maior valor encontrado ocorreu no percolado do Espodossolo, na dose de $700 \mathrm{~m}^{3} \mathrm{ha}^{-1}$, classificando o efluente como água, com nenhum grau de restrição para uso em irrigação.

A redução de SDT, tanto aos 30 quanto aos 60 dias, obedeceu a uma diminuição que pode ser explicada pela textura dos diferentes solos utilizados no experimento, ou seja, o percolado do solo mais arenoso obteve menor taxa de remoção quando comparados os seus valores com os da vinhaça in natura. A taxa de remoção do Argissolo textura argilosa/ arenosa foi maior que a do Espodossolo, porém ainda menor que a do Nitossolo, textura argilosa, corroborando com Sengik et al. (1988) que ressaltam a importância das características do solo na influência dos resultados.

As variáveis SDT e CE obtiveram comportamento praticamente idêntico pois são dois parâmetros altamente correlacionados, corroborando com Lyra et al. (2003), água coletada do lençol freático de área fertirrigada com vinhaça e Ayers \& Westcot (1991), quando interpreta a qualidade de água para irrigação.

Em relação ao $\mathrm{pH}$, os efeitos do solo foram significativos, como: dose, tempo e interação tempo $\times$ taxa de aplicação de vinhaça; as demais interações não o foram. Os maiores $(\mathrm{P} \leq 0,05)$ valores de $\mathrm{pH}$ ocorreram no Nitossolo e Argissolo, que não diferiram entre si nos dois períodos estudados (Figura 1I e J). A adição de vinhaça aos solos diminuiu o pH dos percolados verificando-se, aos 30 dias, diferenças significativas entre solos tratados e não tratados mas depois de determinado período o $\mathrm{pH}$ aumentou, de forma que, aos 60 dias, os percolados de solos tratados indicavam $\mathrm{pH}$ levemente mais elevado que o das
Testemunhas; contudo, sem diferirem estatisticamente. Devido a degradação mais intensa da matéria orgânica após os 30 dias, provocou-se a liberação de eléctrons que reduziram o oxigênio da água e, com isto, diminuíram a concentração do hidrogênio elevando, conseqüentemente, o $\mathrm{pH}$ dos percolados.

De maneira geral, os resultados obtidos mostraram que a aplicação da vinhaça ao solo não trouxe maiores preocupações quanto às questões ambientais. Para os valores estudados, quando se comparam os valores do percolado dos solos tratados com os da vinhaça in natura e a legislação vigente (Brasil, 1986), os solos, apesar de apresentarem diferentes textura e, em conseqüência, diferentes propriedades químicas e físicas, sofreram elevado poder de retenção diminuindo, sobremaneira, a possibilidade deste resíduo causar poluição às águas subsuperficiais até a profundidade estudada. Somado a este fator, também deve ser levada em consideração a diluição, visto que a vinhaça aplicada sofreu diluição na coluna de solo por conta das lâminas de lixiviação aplicadas.

\section{CONCLUSÕES}

1. A DQO do percolado apresentou valores muito inferiores aos da vinhaça in-natura.

2. A DBO dos percolados dos três tipos de solo foi significativamente menor que a da vinhaça in-natura.

3. Os solos tiveram elevado poder de retenção do SDT quando comparados os valores do percolado com os da vinhaça in-natura.

4. A CE no percolado apresentou comportamento semelhante ao da variável SDT, invertendo os valores do Argissolo e do Espodossolo.

5. A vinhaça aplicada com o tempo maior de incubação no solo, elevou o pH do percolado, aproximando-se dos valores da Testemunha.

6. De maneira geral, a aplicação da vinhaça ao solo não favoreceu maiores preocupações quanto as questões ambientais.

\section{LITERATURA CITADA}

APHA - American Public Health Association. Standard methods for the examination of water and wastewater. 15 ed. Washington, D.C.: APHA. AWWA. WPCF, 1995, 1134p.

Ayers, R. S.; Westcot, D. W. A. A qualidade da água na agricultura. Campina Grande: UFPB. 1991. 218p. Estudos FAO: Irrigação e Drenagem, 29.

Brasil. Resolução CONAMA n.20, de 18 de junho de 1986. In: Diário Oficial da União. Distrito Federal, jul. 1986.

Brito, F. L.; Lyra, M. R. C.; Rolim, M. M. Efeito da aplicação de vinhaça em colunas de solo: avaliação do poder de remoção de alguns parâmetros. In: Congresso Brasileiro de Engenharia Agrícola, 32, 2003, Goiania. Resumos... Goiania: SBEA, 2003. CD-Room.

Camargo, O. A.; Berton, R. S.; Valadares, J. M. A. S.; Teófilo Sobrinho, J. Características físicas de solo que recebeu vinhaça. Campinas: Instituto Agronômico de Campinas, 1988, 12p. Boletim Científico, 14 
Camargo, O. A.; Valadares, J. M. A. S.; Geraldi, R. N. Características químicas e físicas de solo que recebeu vinhaça por longo tempo. Campinas: Instituto Agronômico de Campinas, 1983, 30p. Boletim Técnico, 76

Cambuim, F. A. A ação da vinhaça sobre a retenção de umidade, pH, acidez total, acumulação e lixiviação de nutrientes, em solo arenoso. Recife: UFRPE, 1983, 133p. Dissertação Mestrado

Casarini, D. C. P.; Cunha, R. C. A.; Masset Filho, B. Effects of irrigation with vinasse and the dynamics of its constituents in the soil: II Microbiological aspescts. Water Science Technology, v.19, n.8, p.167-176, 1987.

Cunha, R. C. de A.; Costa, A. C. S.; Maset Filho, B.; Casarini, D. C. P. Effects of irrigation with vinasse and the dynamics of its constituents in the soil: I - Physical and chemicals aspects. Water Science and Technology, Colchester, v.19, n.8, p.155-156, 1987.

Ferreira, E. S.; Monteiro, A. O. Efeitos da aplicação da vinhaça nas propriedades químicas, físicas e biológicas do solo. Boletim Técnico. COPERSUCAR, Piracicaba, v.37, p.3-7, 1987.

Gloeden, E.; Cunha, R. C.; Fraccaroli, M. J. B.; Cleary, R. W. The behavior of vinasse constituents in the unsaturated and saturated zones in the Botucatu Aquifer recharge area. São Paulo: CETESB, 1990, 11p.
Ludovice, M. T. F. Estudo do efeito poluente da vinhaça infiltrada em canal condutor de terra sobre o lençol freático. Campinas: UNICAMP, 1997. 117p. Dissertação Mestrado

Lyra, M. R. C. C.; Rolim, M. M.; Silva, J. A. A. Toposseqüência de solos fertigados com vinhaça: contribuição para a qualidade das águas do lençol freático. Revista Brasileira de Engenharia Agrícola e Ambiental, Campina Grande, v.7, n.3, p.523-532, 2003.

Orlando Filho, J.; Zambello Jr., E.; Agujaru, R. Efeito da aplicação prolongada da vinhaça nas propriedades químicas dos solos com cana de açúcar: Estudo exploratório. Stab, Piracicaba, v.1, p.28-33, 1983.

Paganini, W. S. Disposição de esgoto no solo (Escoamento à superfície). São Paulo: AESABESP, 1997. 232p.

RIDESA - Rede Interinstitucional para o Desenvolvimento SucroAlcooleiro. Aspectos ambientais da aplicação da vinhaça no solo. São Paulo: UFSCar, 1994. 67p.

Sengik, E.; Ribeiro, A. C.; Conde, A. R. Efeito da vinhaça em algumas propriedades de amostras de dois solos de Viçosa, MG. Revista Brasileira de Ciência do Solo, Campinas, v.12, n.1, p.11-15, 1988.

Xavier, L. H. Modelos univariado e multivariado para análise de medidas repetidas e verificação de acurácia por meio de simulação. Piracicaba: USP, 2000. 91p. Dissertação Mestrado 